\title{
The Logic of Conditional Belief
}

\author{
Benjamin Eva
}

\section{Forthcoming in Philosophical Quarterly}

\begin{abstract}
The logic of indicative conditionals remains the topic of deep and intractable philosophical disagreement. I show that two influential epistemic norms - the Lockean theory of belief and the Ramsey test for conditional belief - are jointly sufficient to ground a powerful new argument for a particular conception of the logic of indicative conditionals. Specifically, the argument demonstrates, contrary to the received historical narrative, that there is a real sense in which Stalnaker's semantics for the indicative did succeed in capturing the logic of the Ramseyan indicative conditional.
\end{abstract}

\section{$\S 1:$ Introduction}

What is the propositional content expressed by an indicative conditional sentence of the form 'If $\phi$, then $\psi$ '? According to the material conditional analysis of the indicative, 'If $\phi$, then $\psi$ ' expresses the truth-functional proposition $\phi \supset \psi={ }_{d f} \neg \phi \vee \psi$. On the strict conditional analysis, 'If $\phi$, then $\psi$ ' expresses the modal proposition $\square(\phi \supset \psi)$. And on the Stalnaker semantics, 'If $\phi$, then $\psi$ ' expresses the proposition $\phi \Rightarrow \psi={ }_{d f}$ ' $\psi$ is true at the closest world at which $\phi$ is true (the closest $\phi$-world is a $\psi$-world)'. ${ }^{1}$ Debates between advocates of these competing conceptions of the truth-conditions of the indicative tend to focus largely on the comparative plausibility of each account's view of conditional inference ${ }^{2}$ i.e. on which conditional inferences they class as (in)valid (where validity is understood in the standard way, as necessary truth preservation). To illustrate, consider the accounts' verdicts

\footnotetext{
${ }^{1}$ Although the Stalnaker semantics is most commonly employed to model the propositional content of subjunctive conditionals, Stalnaker explicitly contested that his semantics captures the content of conditionals phrased in both the subjunctive and indicative moods (see e.g. Stalnaker (1968)). On Stalnaker's account, the distinction between subjunctive and indicative conditionals is accounted for by stipulating that the conception of 'closeness between worlds' at play in the evaluation of subjunctives is different to the conception used for indicatives.

${ }^{2}$ Of course, there are many alternative semantic theories of the indicative conditional, several of which eschew the assumption that the indicative has determinate truth-conditions. Here, I restrict my attention to truth-conditional semantics. In particular, the three truth-conditional semantics mentioned above (arguably the most influential in the literature) will be sufficient for the expository purposes to which they are applied. For canonical expositions and defences of the material conditional, strict conditional and Stalnaker semantics for the indicative, see Jackson (1979), Lewis (1918) and Stalnaker (1968), respectively.
} 


\begin{tabular}{|c|c|c|c|}
\hline MP & $\phi, \phi \rightarrow \psi \vdash \psi$ & MT & $\neg \psi, \phi \rightarrow \psi \vdash \neg \phi$ \\
\hline RT & $\phi \rightarrow \psi,(\phi \wedge \psi) \rightarrow \xi \vdash \phi \rightarrow \xi$ & ID & $\vdash \phi \rightarrow \phi$ \\
\hline $\mathrm{CC}$ & $\phi \rightarrow \psi, \phi \rightarrow \xi \vdash \phi \rightarrow(\psi \wedge \xi)$ & CA & $\phi \rightarrow \xi, \psi \rightarrow \xi \vdash(\phi \vee \psi) \rightarrow \xi$ \\
\hline CMon & $\phi \rightarrow \xi, \phi \rightarrow \psi \vdash(\phi \wedge \psi) \rightarrow \xi$ & $\mathrm{CSO}$ & $\phi \rightarrow \psi, \psi \rightarrow \phi, \phi \rightarrow \xi \vdash \psi \rightarrow \xi$ \\
\hline CONT & $\phi \rightarrow \psi \vdash \neg \psi \rightarrow \neg \phi$ & $\mathrm{CCO}$ & $\psi \rightarrow \xi, \xi \rightarrow \psi, \phi \rightarrow \psi \vdash \phi \rightarrow \xi$ \\
\hline HS & $\phi \rightarrow \psi, \psi \rightarrow \xi \vdash \xi$ & SA & $\phi \rightarrow \xi \vdash(\phi \wedge \psi) \rightarrow \xi$ \\
\hline $\mathbf{C N}_{1}$ & $\neg(\phi \rightarrow \psi) \vdash(\phi \rightarrow \neg \psi)$ & $\mathrm{CS}$ & $\phi \wedge \psi \vdash \phi \rightarrow \psi$ \\
\hline $\mathrm{CN}_{2}$ & $\phi \rightarrow \neg \psi \vdash \neg(\phi \rightarrow \psi)$ & OIF & $\phi \vee \psi \vdash \neg \phi \rightarrow \psi$ \\
\hline RCR & $(\psi \wedge \xi) \supset \gamma, \phi \rightarrow \psi, \phi \rightarrow \xi \vdash \phi \rightarrow \gamma$ & RCM & $\psi \supset \xi, \phi \rightarrow \psi \vdash \phi \rightarrow \xi$ \\
\hline RCEA & $\phi \equiv \psi, \phi \rightarrow \xi \vdash \psi \rightarrow \xi$ & RCEC & $\psi \equiv \xi, \phi \rightarrow \psi \vdash \phi \rightarrow \xi$ \\
\hline $\mathrm{TC}$ & $\psi \vdash \phi \rightarrow \psi$ & NA & $\neg \phi \vdash \phi \rightarrow \psi$ \\
\hline & $\begin{array}{cc}\square \supset+\Rightarrow & \supset+\square \supset \\
\square & \square\end{array}$ & & $\begin{array}{c}\Rightarrow+\square \supset \\
\square\end{array}$ \\
\hline
\end{tabular}

Figure 1: 22 Prospective Conditional Inference Rules, and their validity profiles according to $\supset, \square \supset$ and $\Rightarrow$ (the colour shadings indicate which rules are validated by which semantics).

on the validity of the important conditional inference rules summarised in Figure 1 (where $\phi \rightarrow \psi$ always denotes the indicative conditional 'If $\phi$ then $\psi$ ').

Given the substantive disagreement (exhibited in Figure 1) between the conditional logics yielded by the three semantics, the question now arises: "which of these competing conceptions of the logic of the indicative conditional is the most plausible?' - or, alternatively, 'which conditional inferences should we expect to be validated by a prospective semantics for the indicative?'. Despite prolonged and spirited debate on the logical status of many individual conditional inferences, ${ }^{3}$ this question remains the subject of much controversy. This being the case, it remains difficult to adjudicate between rival semantics for the indicative. How can we decide which semantics is best if we don't know which conditional inferences we want to be validated?

The aim of this paper is to develop a new approach to the question "which conditional inferences should we expect to be validated by a prospective semantics for the indicative?'. Roughly summarised, this new approach will begin by assuming some widely accepted and independently plausible epistemic norms regarding the nature of rational belief and its relation to logical inference, before proceeding to demonstrate that the combination of those norms commits one to a specific conception of the logic of indicative conditionals. More specifically, the plan is as follows. I begin (§2) by recalling two influential epistemic norms regarding rational belief: the Lockean theory of belief and the Ramsey test for conditional belief. I then describe (recalling work by Douven (2015))

\footnotetext{
${ }^{3}$ In the contemporary literature, there are extended debates regarding the validity of i.e. MP (McGee (1985), Hartmann and Stern (forthcoming)), MT (Yalcin, 2012), CONT (Adams, 1975) and HS (Adams (1975), Starr (unpublished)), for example.
} 
how the two can naturally be combined to provide a Lockean theory of conditional belief. In $\S 3$, I turn to defending a very weak consistency norm for rational belief. $\S 4$ is where the rubber hits the road. Here, I show how the Lockean theory of conditional belief, when combined with the weak consistency norm for rational belief, provides a new methodology for determining which conditional inference rules are valid. As it turns out, the set of conditional inferences that are validated by this methodology coincides exactly with the set of inferences validated by Stalnaker's semantics for the indicative, and therby provides an independent epistemic justification for Stalnaker's conception of the logic of indicative conditionals.

\section{§2: The Lockean Theory of Conditional Belief}

\section{§2.1: The Lockean Theory of Belief}

Under what circumstances is it rational for an agent to believe a proposition $p$ ? According to the influential Lockean theory of belief, the answer to this question is best articulated in terms of numerically graded confidence, or 'credence'. Specifically, all advocates of the Lockean theory defend some variation of the following 'Lockean thesis'.

The Lockean Thesis (LT:) [I]t is epistemically rational for us to believe a proposition just in case it is epistemically rational for us to have a sufficiently high degree of confidence in it. (Foley 1992: 111)

The driving intuition is this. We should believe those propositions in which we are highly confident, disbelieve those propositions in which we have low confidence, and suspend judgement about those propositions for which we have a middling degree of confidence. Of course, to make all of this precise we need to specify exactly what is meant by 'high/low confidence'. This is normally done by assuming that there exists some threshold $t$ (called the 'Lockean threshold') such that it is rational to believe a proposition $\phi$ if and only if one's credence in $\phi$ is at least $t$, i.e. $c(\phi) \geq t$. Assuming that the credences of a rational agent are always probabilistic, we can suppose that $t \in[0,1]$. It is also intuitively compelling to require that both (i) $t<1$, and (ii) $t>\frac{1}{2}$, are satisfied. ${ }^{4}$ (i) encodes

\footnotetext{
${ }^{4}$ While (ii) seems to be universally accepted, there are some authors who endorse a version of LT on which $t=1$ is a constant requirement, i.e. on which belief is equated with certainty of a particular kind (see e.g. Clarke (2013)).
} 
the requirement that rational belief should not be identified with certainty, since I rationally believe many things of which I am not certain (that it's currently raining somewhere in Cornwall, for instance). (ii) encodes the requirement that it is never rational for an agent to believe $\phi$ without being more confident in $\phi$ than they are in $\neg \phi$. If (ii) were violated, it would be rationally admissible to believe of a fair coin both that it will land heads, and also that it will land tails, for example. One simple and important point to note here is that once LT has been spelled out in terms of a Lockean threshold $t \in\left(\frac{1}{2}, 1\right)$, we can equate disbelief in $\phi$ (belief in $\neg \phi$ ) with having credence $c(\phi) \leq 1-t$, since this is equivalent to having credence $c(\neg \phi) \geq t$.

The Lockean theory of belief, formalised in this probabilistic interpretation of LT, will be one of the basic operating premises of the proceeding analysis. In the interest of concision, I outsource the defence of this premise to i.e. Foley (2000), and simply assume LT as a fundamental normative constraint on the relationship between the beliefs and credences of all rational agents.

\section{§2.2: The Ramsey Test for Conditional Belief}

Having specified a general background theory of categorical belief and its relationship to probabilistic credence, I turn now to specifying my preferred theory of conditional belief, i.e. a theory that specifies the conditions under which it is rational to believe an indicative conditional of the form 'If $\phi$ then $\psi$ '. By far the most widely employed theory of conditional belief is given by the 'Ramsey Test', which is commonly articulated in something like the following form,

The Ramsey Test for Conditional Belief $\left(\mathbf{R T}_{0}\right)$ : It is rational to believe an indicative conditional of the form 'If $\phi$ then $\psi$ ' if and only if it would be rational to believe $\psi$ upon learning the truth of $\phi .56$

$\mathrm{RT}_{0}$ reduces the (synchronic) rationality norms for conditional belief to the (diachronic) ratio-

\footnotetext{
${ }^{5}$ Of course, it is assumed here that the relevant learning experience is one in which $\phi$ is the only thing that is learned. I also follow orthodoxy in assuming that $\phi \rightarrow \psi$ can only be meaningfully considered when $\phi$ is not known to be false.

${ }^{6}$ Although the historical subtleties are not of great relevance here, it is important to note that the correct interpretation of Ramsey's original comments on conditional belief is the subject of significant controversy. However, the formulation of $\mathrm{RT}_{0}$ given here is very close to some of the most common interpretations from the literature. For example, Stalnaker (1968) formulates the Ramsey test in the following way,
}

This is how to evaluate a conditional. First, add the antecedent (hypothetically) to your stock of beliefs; second, make whatever adjustments are required to maintain consistency (without modifying the hypothetical belief in the antecedent); finally, consider whether or not the consequent is then true. (Stalnaker, 1968: 101) 
nality norms for learning. In so doing, it provides a natural explanation of the close relationship between conditional judgement and suppositional judgement, and allows us to derive concrete rules for conditional belief formation from an account of rational learning dynamics. Again, there already exists a vast literature defending $\mathrm{RT}_{0}$ as the basic norm of conditional belief (see e.g. Bradley (2007), Hanson (1992)), and it would only muddy the waters to rehash those debates here. I am happy to simply accept $\mathrm{RT}_{0}$ as a basic premise in what follows.

But before moving on, I need to augment this standard interpretation of the Ramsey test with an important additional clause. Specifically, it should be noted that in the current formulation, $\mathrm{RT}_{0}$ specifies the conditions under which it is rational to believe an indicative conditional, but remains silent regarding the conditions under which it is rational to disbelieve an indicative. Since disbelief will play a central role in what follows, I will add an extra clause to $\mathrm{RT}_{0}$ specifying conditions for rational disbelief of indicative conditionals. Intuitively, there are two natural ways to extend $\mathrm{RT}_{0}$ to cover the norms for disbelief, namely

(i) It is rational to disbelieve 'If $\phi$ then $\psi$ ' if and only if it would be rational to disbelieve $\psi$ upon learning the truth of $\phi$.

(ii) It is rational to disbelieve 'If $\phi$ then $\psi$ ' if and only if it would be rational either to disbelieve $\psi$ or to suspend judgement about $\psi$ upon learning the truth of $\phi$.

Clearly, (i) poses much stricter conditions for disbelief than (ii) does. In fact, it is easily observed that, when combined with $\mathrm{RT}_{0}$, (ii) entails that it is never rationally permissible to suspend judgement about an indicative conditional, which is an absurd conclusion. As I'm writing this, I neither believe nor disbelieve the conditional 'If it rains tomorrow, it will be sunny the next day', and I do not feel myself to be irrational in this regard. So it seems clear that (i) is the most plausible way to naturally extend $\mathrm{RT}_{0}$ to a joint theory of conditional belief and conditional disbelief. Thus, the second main premise of my argument will be

The Ramsey Test for Conditional Belief and Disbelief (RT): It is rational to believe an indicative conditional of the form 'If $\phi$ then $\psi$ ' if and only if it would be rational to believe $\psi$ upon learning the truth of $\phi$. Conversely, it is rational to disbelieve 'If $\phi$ then $\psi$ ' if and only if it would be rational to disbelieve $\psi$ upon learning the truth of $\phi$. 


\section{§2.3: The Lockean Ramsey Test}

Now that we've assumed LT and RT as our background theories of categorical and conditional belief, respectively, we can combine them to obtain a distinctively Lockean formulation of RT. To see this, note first that there exists a single rationally privileged learning rule for agents with probabilistic credences, namely Bayesian conditionalisation. ${ }^{7}$ Accordingly, we can give a Lockean rephrasing of the claim 'It is rational to believe $\psi$ upon learning the truth of $\phi$ ' as 'It is rational to have sufficiently high credence in $\psi$ after learning $\phi$ ', or, more formally, ' $c(\psi \mid \phi) \geq t$ '. ${ }^{8}$ This rephrasing yields the following Lockean formulation of RT,

The Lockean Ramsey Test for Conditional Belief and Disbelief (LRT): It is rational to believe an indicative conditional of the form 'If $\phi$ then $\psi$ ' if and only if $c(\psi \mid \phi) \geq t$. Conversely, it is rational to disbelieve 'If $\phi$ then $\psi$ ' if and only if $c(\psi \mid \phi) \leq 1-t$

LRT is very closely analogous to Douven's (2015) 'Qualitative Adam's Thesis', the only differences being that (i) while LRT is formulated in terms of rational belief, Douven phrases the Qualitative Adam's Thesis in terms of rational acceptability, and (ii) Douven does not specify conditions under which it is rational to disbelieve conditionals.

In what follows, I will assume LT, RT and LRT as universal norms of ideal rationality. ${ }^{9}$ I turn now to introducing one last epistemic norm which, when combined with LT, RT and LRT, will be sufficient to ground the argument for a specific conception of the logic of indicative conditionals. Note that I use the term 'Lockean agent' to refer to agents that have probabilistic credences and form qualitative beliefs in accordance with LT, RT and LRT (for some fixed threshold $t$ ).

\footnotetext{
${ }^{7}$ I assume Bayesian conditionalisation as a rule of ideal rationality throughout. However, I do not state this as an explicit premise of my arguments, since the rule is so widely accepted and standardly assumed as part of the cognitive furniture of rational agents.

${ }^{8}$ Where $c(\psi \mid \phi)$ denotes the posterior credence in $\psi$ after conditionalising on $\phi$ (the conditional probability of $\psi$ given $\phi)$. Note that Shear and Fitelson (2018) and Eva, Shear and Fitelson (unpublished) have recently surveyed the structure of the qualitative belief change mechanism defined by the combination of LT and Bayesian conditionalisation.

${ }^{9}$ Although of course LRT is not really an additional assumption, since it is entailed by LT and RT modulo Bayesian conditionalisation.
} 


\section{§3: A Weak Consistency Norm for Rational Belief}

Here are two prospective epistemic norms that are widely taken as partially constitutive of the notion of rational belief (see i.e. Leitgeb (2017)).

Consistency of Rational Belief (CON): The beliefs of a rational agent should always be logically consistent.

Closure of Rational Belief (CLO): The beliefs of a rational agent should always be closed under logical entailment.

It is well known that the Lockean conception of belief, enshrined in LT, is in tension with both of these norms. This is most easily illustrated via Kyburg's (1961) famous lottery paradox.

A fair, 1-winner, million ticket lottery is held, meaning that for any ticket number $i$, the chance of $i$ being the winning ticket is $\frac{1}{1,000,000}$. So for any ticket number $i$, a rational agent will have credence $c\left(w_{i}\right)=\frac{1}{1,000,000}$ in $i$ 's being the winning ticket. So for each ticket number $i$, a rational agent will disbelieve the proposition $w_{i}={ }_{d f}$ 'ticket $i$ is the winning ticket' (assuming $t \leq \frac{999,999}{1,000,000}$ ), ${ }^{10}$ i.e. they will believe the proposition $\neg w_{i}=$ 'ticket $i$ is not the winning ticket'. But by stipulation, the lottery is a 1-winner lottery, meaning that one of the million tickets must be the winning ticket. So the chance of the disjunction ' $w_{1} \vee w_{2} \vee \ldots \vee w_{1,000,000}$ ' being true is 1 . So a rational agent will have credence $c\left(w_{1} \vee w_{2} \vee \ldots \vee w_{1,000,000}\right)=1$ and will therefore believe the disjunction $w_{1} \vee w_{2} \vee \ldots \vee w_{1,000,000}$ for any specification of the Lockean threshold. So for each $i$, the agent will believe $\neg w_{i}$, whilst simultaneously believing the disjunction $w_{1} \vee w_{2} \vee \ldots \vee w_{1,000,000}$. But this is inconsistent. There is no possible world at which all of those beliefs are true. ${ }^{11}$

In some quarters, the fact that the Lockean theory conflicts so straightforwardly with CON and CLO is taken to constitute a decisive refutation of the theory. ${ }^{12}$ Conversely, advocates of the Lockean theory typically construe this conflict as a demonstration that CON and CLO should be abandoned as universal norms of ideal rationality (see i.e. Foley (2000)). Given that I am assuming

\footnotetext{
${ }^{10}$ Of course, one could demand a higher threshold, but the paradox is easily reconstructed by considering an even larger lottery.

${ }^{11}$ It's easy to see that the agent's beliefs also violate CLO in this case.

${ }^{12}$ For example, Leitgeb (2017) takes the violation of CON and CLO by LT as the primary motivation for the development of his 'Stability Theory of Belief'.
} 
the Lockean theory of belief as a premise for the present analysis, I am of course compelled to take the Lockean's side here and reject both CON and CLO as fundamental edicts of ideal rationality. Specifically, I contend that the epistemic state of the Lockean agent considering the lottery paradox is perfectly justifiable and therefore rational. If this is right (as many authors claim), then we have strong reason to abandon CON and CLO.

However, although I am happy to reject $\mathrm{CON}$ as a fully general constraint on the beliefs of rational agents, I do still think that there is some role to be played by consistency norms for rational belief. To see why, consider the following variation of the lottery paradox. Instead of a fair, 1-winner, million ticket lottery, imagine now a fair, 1-winner, 2-ticket lottery. Now imagine I tell you that I believe ticket 1 will lose, and I believe ticket 2 will lose, but I also believe that one of the two tickets will win, i.e. I simultaneously believe $\neg w_{1}, \neg w_{2}$ and $w_{1} \vee w_{2}$. I think it is clear that there is something fundamentally strange and irrational about my beliefs in this case, in a sense that does not apply to my beliefs in the million ticket case. It is hard to imagine how I could ever be justified in holding all three of those beliefs at the same time. For, the fair, 1-winner, two ticket lottery is essentially the same situation as a fair coin toss, and we certainly think it's never justifiable to simultaneously believe of a fair coin both that it will land heads and that it will land tails. In contrast, in the million ticket case, it seems that all of my individual beliefs are perfectly well justified, and that it is perfectly reasonable for me to hold them all simultaneously, even though they are inconsistent.

This suggest that there is one kind of consistency violation (the kind that occurs in the million ticket lottery example) that is sometimes rationally permissible, and there is another kind (the kind that occurs in the two ticket lottery example) that is never rationally permissible. To make this distinction precise, note that in the million ticket case, the agent believes all 1,000,000 premises of the following valid inference whilst disbelieving the conclusion.

$$
\neg w_{1}, \neg w_{2}, \ldots ., \neg w_{1,000,000} \vdash \neg w_{1} \wedge \neg w_{2} \wedge \ldots \wedge \neg \neg w_{1,000,000}
$$

I follow the Lockean orthodoxy in contesting that this can sometimes be rationally permissible, i.e. it can be rationally permissible to believe all the premises of a valid million premise inference whilst disbelieving the conclusion.

Meanwhile, in the two-ticket case, the agent believes both the premises of the following valid inference whilst disbelieving the conclusion.

$$
\neg w_{1}, \neg w_{2} \vdash \neg w_{1} \wedge \neg w_{2}
$$


I've argued that we should never think that this is rationally permissible, i.e. it is never rationally permissible to believe all the premises of a valid two premise inference whilst disbelieving the conclusion. Call this claim the 'weak consistency norm'.

Weak Consistency Norm (WCON): A rational agent should never believe all the premises of a valid two (or less) premise inference whilst disbelieving the conclusion.

I take it that WCON prohibits only the most egregious and obviously problematic forms of inconsistency. One might be tempted to posit a much stronger consistency norm, on which it is always irrational to believe the premises of an $n$ (or less) premise valid inference whilst disbelieving the conclusion for $n>>2$. And that's fine with me. Maybe we should draw the line at 10,000 premises, or 1,000 , or 100 , or 10 , or 5 . But wherever we draw the line, it certainly shouldn't be below $2 .^{13}$ In this sense, WCON is the weakest logical consistency norm for rational belief apart from the direct injunction that one should never believe a contradiction. ${ }^{14}$

The idea then is that inconsistencies of the type that involve WCON violations are never rationally permitted. However, if the set of propositions that is used to generate the inconsistency is sufficiently large (as in the 1,000,000 ticket case), then the inconsistency may be permissible. This can be seen as a formalisation of Foley's contention that

[A]lthough inconsistency in a very large...set of propositions need not have much negative epistemic significance, inconsistency among a very small...set does. The latter precludes the possibility that it is rational for you to believe each and every proposition in the set, but the former need not. (Foley, 1991: 6)

Similar sentiments are expressed by e.g. Knight (2002), Kyburg (1961) and Sorensen (2006), who writes (summarising Kyburg)

[A]lthough the dull pressure of joint inconsistency is tolerable when diffusely distributed

\footnotetext{
${ }^{13}$ For what it's worth, I think this looks like a paradigmatic instance of normative vagueness. It's definitely rationally permissible to be inconsistent in the 1,000,000 premise case, and its definitely impermissible in the 2 premise case. Somewhere between these two cases, things get murky, and it seems unlikely that we can draw a boundary between the permissible and impermissible cases in a principled and plausible manner. Similarly, it is presumably morally inadmissible to leave a 1 month old baby alone without adult supervision for, say one hour. At the other end of the spectrum, it is certainly permissible to leave a 16 year old alone without supervision for one hour. But things gets fuzzy somewhere in the middle (for example, for a nine year old).

${ }^{14}$ An important clarification: WCON does not require rational agents to believe the conclusions of instances of valid two premise inferences whose premises they believe. It only requires them not to simultaneously believe the premises whilst also disbelieving the conclusion.
} 
over a large set of propositions, the pain of contradiction becomes unbearable as the set gets smaller. (Sorensen, 2006: §4)

WCON says that wherever we identify the point at which 'the pain of contradiction becomes unbearable', it should certainly be before the point at which agents are led to believe the premises of a valid 2-premise inference whilst disbelieving the conclusion.

At this stage, one might wonder whether we can ever ensure that agents who abide by the probabilistic formalisation of LT will generally satisfy WCON. To answer this question, the following definition will be helpful.

Definition 1: For any inference rule $R$ and any admissible value $t \in\left(\frac{1}{2}, 1\right)$ of the Lockean threshold, say that $R$ is ' $t$-valid' if and only if it is impossible for a Lockean agent with Lockean threshold t to believe R's premises whilst disbelieving R's conclusion.

The following result is due to Hawthorne and Bovens (1991). ${ }^{15}$

Proposition 1 Let $R$ be a 2-premise inference rule of classical truth functional logic. If $R$ is valid, then $R$ is t-valid for all and only values of $t>\frac{2}{3}$. If $R$ is invalid then $R$ is not $t$-valid for any admissible value of $t$.

And Proposition 1 entails the following important corollary.

Corollary 2 In the context of classical truth functional logic, a Lockean agent is guaranteed to satisfy WCON if and only if the Lockean threshold satisfies the inequality $t>\frac{2}{3} \cdot 16$

Corollary 2 shows that advocates of the Lockean thesis can indeed posit WCON as a universal rule of ideal rationality in the context in which only ordinary truth functional propositions are considered. As long as the Lockean threshold for belief is set above $\frac{2}{3}$, agents that adhere to LT will indeed be guaranteed to satisfy WCON (with respect to classical truth functional logic). Of course, this provides good reason to stipulate that the Lockean threshold for belief should never be allowed to fall below $\frac{2}{3} \cdot{ }^{17}$ In what follows, I will assume WCON as the final piece of the normative

\footnotetext{
${ }^{15}$ Actually, Hawthorne and Bovens prove a slightly different result (showing that $n$-ary conjunction introduction is $t$-valid for all and only $t>\frac{n}{n+1}$ ), but this follows as an easy corollary.

${ }^{16}$ Note that since we are restricting to truth-functional logic, a Lockean agent here can be understood as an agent with probabilistic credences that forms beliefs in accordance with LT. Since we are ignoring indicative conditionals, RT and LRT play no role at this stage.

${ }^{17}$ This holds regardless of whether one thinks the Lockean threshold should be allowed to vary with context.
} 
jigsaw that already contains LT, RT and LRT and eventually yields a coherent sketch of the logic of indicative conditionals.

\section{§4: The Logic of Conditional Belief}

Enough with the detours. It's time to return to the basic motivating question of the article: 'which conditional inferences should we expect to be validated by a prospective semantics for the indicative conditional?'. To answer this question, I'll rely on the epistemic norms LT, RT, LRT and WCON described above. In fact, my answer requires only one additional premise for its derivation, namely

The Hopeful Premise (HOPE): There always exists an epistemic strategy such that agents who follow that strategy are guaranteed not to violate any norms of ideal rationality.

The idea behind HOPE is simple - it should be possible for agents to reason in a way which guarantees that they don't end up violating any fundamental epistemic norms. In the present context, HOPE's salient implication is that it should be possible for agents to reason in a way which ensures that they don't end up violating any of LT, RT, LRT and WCON.

Why is it legitimate for me to assume HOPE as a premise for my argument? That's a big question that goes far beyond the scope of the present enquiry. But I should note that principles like HOPE play a central role in the theory of normativity more generally. For example, one of the most influential criticisms of normative ethical theories that build on deontological principles like 'Don't lie' and 'Protect your friends from harm' is that it is sometimes impossible to act in a way that simultaneously accords with all of those principles, i.e. that deontological theories of normative ethics (often) entail that there does not exist a universal strategy such that agents who follow the strategy are guaranteed not to violate any ethical norms. ${ }^{18}$ In contrast, I take it that one of the primary theoretical virtues of a naive Utilitarian conception of normative ethics is that it explicitly identifies a strategy which guarantees accordance with the sole fundamental ethical norm of attempting to maximise total utility wherever possible (namely the strategy of attempting to maximise total utility wherever possible). So the driving intuition behind HOPE is a familiar one, namely that it should always be possible for an agent to perform in a way which ensures accordance with all relevant norms. If HOPE were to fail, then there would always be some risk that, however

\footnotetext{
${ }^{18}$ Recall Kant's axe murderer.
} 
an agent behaves, they will end up violating a basic norm. And that would be deeply unsatisfactory. Only a cruel game would have rules which are rigged in that way. Of course, none of this constitutes a rigorous justification of HOPE. For all we know, rationality could well be a cruel game. Maybe there's no way to ensure constant accordance with all the laws of ideal rationality. But I, like many others, am happy to operate under the assumption that rationality is not a cruel game, and that there exist reasoning strategies which do keep one from violating basic epistemic norms with perfect reliability. ${ }^{19}$

At any rate, I will now assume HOPE as the final premise in my reply to the question 'which conditional inferences should we expect to be validated by a prospective semantics for the indicative conditional?'. The other premises are given by LT, RT and LRT, where the reference to 'Lockean agents' in the statement of WCON is now understood to entail that the relevant agents form conditional beliefs in accordance with LRT, and the phrase 'valid inferences' is understood to include the valid inferences of indicative conditional logic.

Here's the first part of the argument.

(Premise 1) LT, RT, LRT and WCON are all norms of ideal rationality.

(Premise 2) HOPE

(3) If Premise 1 and Premise 2 are both true, then all valid 2 (or less) premise conditional inferences are $t$-valid for some admissible values of the Lockean threshold.

(4) So all valid 2 (or less) premise conditional inferences are $t$-valid for some admissible values of the Lockean threshold (from 1, 2 and 3).

The first thing to note is that 4 is logically entailed by the conjunction of (1), (2) and (3) via modus ponens. ${ }^{20}$ Thus, since we're assuming the truth of premises (1) and (2), we only need to establish (3) in order to derive (4).

To establish (3), reason by contradiction and assume it is false. Since we've assumed the truth of premises (1) and (2), this entails that there exists a valid 2 (or less) premise conditional inference $R$ that is not $t$-valid for any admissible value of the Lockean threshold. Now imagine that an agent $A$ reasons in accordance with LT, RT and LRT, and let $t$ denote $A$ 's Lockean threshold for belief. Since $R$ is not $t$-valid, it is possible for $A$ to believe all $R$ 's premises whilst simultaneously disbelieving

\footnotetext{
${ }^{19}$ For what it's worth, I think that this assumption is implicit in the way that many philosophers conceive of the very project of normative epistemology.

${ }^{20}$ Where of course, the 'If...then' construction in (3) denotes a material conditional, not an indicative conditional (assuming the two don't turn out to be the same).
} 
$R$ 's conclusion. And since $R$ is a valid inference with $\leq 2$ premises, this entails that it is possible for $A$ to violate WCON. Thus, however $A$ chooses their threshold for belief, it is possible for them to violate WCON. So there is no reasoning strategy that $A$ can adopt which guarantees that they simultaneously satisfy all of LT, RT, LRT and WCON. But these are all laws of ideal rationality. So it is entailed by HOPE that there should exist a reasoning strategy which guarantees the simultaneous satisfaction of all four norms. Contradiction. So (3) must be true.

One loophole that one might try to exploit against the preceding argument for (3) is the following. I showed that the existence of a valid 2 (or less) premise conditional inference $R$ that is not $t$-valid for any admissible value of the Lockean threshold entails that it will sometimes be the case that an agent who reasons in accordance with LT, RT and LRT violates WCON. That is to say, they can have probabilistic credences which lead them, via LT/RT/LRT, to believe the premises of an instance of $R$ whilst disbelieving the conclusion. Now, one might say that it is still possible for the agent to ensure that they don't violate WCON. All they need to do is make sure that they don't end up adopting those probabilistic credences which, via LT/RT/LRT, lead them to believe the premises of an instance of $R$ whilst disbelieving the conclusion, i.e. for whatever value of $t$ they adopt at a time, they should make sure not to stray into a region of credal space that commits them to a WCON violation. If they do that, then they are, by definition, guaranteed not to violate WCON. But this is not a plausible response. It requires that, for any fixed value of $t$, there exists a set of probabilistic credence functions that the agent is rationally prohibited from adopting. And that seems deeply strange. What if the evidence the agent obtains leads them, via basic epistemic norms such as the Principal Principle or Bayesian Conditionalisation, to adopt credences in the prohibited region of credal space? If the agent changes their credences to avoid a WCON violation, then they will end up violating these other epistemic norms. So they will still fail to satisfy the full gamut of relevant epistemic norms. So this strategy for ensuring the simultaneous satisfaction of LT, RT, LRT and WCON still leads us into contradicting HOPE in the end.

Thus, we have very good reason to accept (3) and, with it, (4). And (4) says something very substantive about indicative conditional logic. In particular, it says that for any valid conditional inference rule $R$ with 2 (or less) premises, there exist admissible values of $t$ for which $R$ is $t$-valid. So if $R$ is not $t$-valid for any admissible values of $t$, then $R$ can't be a valid rule of conditional inference. So, let's check which of the conditional inference rules summarised in Figure 1 are $t$-valid for which values of $t$ (note that $r^{-1}=\frac{500}{809} \sim 0.618$ denotes the inverse of the golden ratio). ${ }^{21}$

\footnotetext{
${ }^{21}$ This is not the first time that $r^{-1}$ has shown up as a theoretically significant threshold for the Lockean theory. Shear
} 
Theorem 3 The t-validity profiles of the inference rules summarised in Figure 1 are given by the table in Figure 2. (Proof in Appendix)

\begin{tabular}{|c|c|c|c|c|}
\hline & $\forall t \in\left(\frac{1}{2}, 1\right)$ & $\forall t \in\left(r^{-1}, 1\right)$ & $\forall t \in\left(\frac{2}{3}, 1\right)$ & $\exists t \in\left(\frac{1}{2}, 1\right)$ \\
\hline CS & $\checkmark$ & $\checkmark$ & $\checkmark$ & $\checkmark$ \\
\hline CN $_{1}$ & $\checkmark$ & $\checkmark$ & $\checkmark$ & $\checkmark$ \\
\hline CN $_{2}$ & $\checkmark$ & $\checkmark$ & $\checkmark$ & $\checkmark$ \\
\hline ID & $\checkmark$ & $\checkmark$ & $\checkmark$ & $\checkmark$ \\
\hline MP & $\times$ & $\checkmark$ & $\checkmark$ & $\checkmark$ \\
\hline MT & $\times$ & $\checkmark$ & $\checkmark$ & $\checkmark$ \\
\hline RT & $\times$ & $\checkmark$ & $\checkmark$ & $\checkmark$ \\
\hline CSO & $\times$ & $\checkmark$ & $\checkmark$ & $\checkmark$ \\
\hline CMon & $\times$ & $\checkmark$ & $\checkmark$ & $\checkmark$ \\
\hline CA & $\times$ & $\checkmark$ & $\checkmark$ & $\checkmark$ \\
\hline CC & $\times$ & $\times$ & $\checkmark$ & $\checkmark$ \\
\hline CONT & $\times$ & $\times$ & $\times$ & $\times$ \\
\hline HS & $\times$ & $\times$ & $\times$ & $\times$ \\
\hline SA & $\times$ & $\times$ & $\times$ & $\times$ \\
\hline CCO & $\times$ & $\times$ & $\times$ & $\times$ \\
\hline NA & $\times$ & $\times$ & $\times$ & $\times$ \\
\hline TC & $\times$ & $\times$ & $\times$ & $\times$ \\
\hline OIF & $\times$ & $\times$ & $\times$ & $\times$ \\
\hline RCM & $\times$ & $\times$ & $\times$ & $\times$ \\
\hline RCR & $\times$ & $\times$ & $\times$ & $\times$ \\
\hline RCEA & $\times$ & $\times$ & $\times$ & $\times$ \\
\hline RCEC & $\times$ & $\times$ & $\times$ & $\times$ \\
\hline
\end{tabular}$+\square+\Rightarrow$

Figure 2: 22 Prospective Conditional Inference Rules, and their full t-validity profiles.

Combined with (4) above, Theorem 3 yields some very strong verdicts on the logic of the indicative conditional. Specifically, it entails that rules such as SA, NA, CONT and HS are all invalid, since they are not $t$-valid for any value of $t \in\left(\frac{1}{2}, 1\right)$. In contrast, rules like MP, MT and CA are all $t$-valid for any value of $t>r^{-1}$, and so (4) is consistent with their validity. Since i.e. SA, NA, CONT and HS are all valid inferences according to the material conditional semantics, we can now conclude that the material conditional semantics cannot be correct. It gives the wrong logic for the indicative. Similarly, since CONT, HS and SA are all valid on the strict conditional semantics, we can conclude

and Fitelson (2018) showed that whether or not $t>r^{-1}$ holds is a crucial factor in determining which AGM belief revision postulates are satisfied by Lockean agents that update their credences by conditionalisation. And one of the technical results summarised in the table below is actually closely related to a central result of Shear and Fitelson (2018). Specifically, the fact that MP is $t$-valid if and only if $t>r^{-1}$ is a significant generalisation of Shear and Fitelson's observation that Lockean agents satisfy the 'very weak preservation' principle if and only if $t>r^{-1}$. 
that the strict conditional gets the logical properties of the indicative wrong, and therefore must be rejected.

In stark contrast, it can be seen that the Stalnaker semantics validates all those inference rules which are $t$-valid for all values of $t>\frac{2}{3}$ and invalidates all those rules that are not $t$-valid for any plausible values of $t .^{22}$ This suggests that the conditional logic defined by the Stalnaker semantics is the strongest conditional logic one can posit for the indicative whilst accepting LT, RT, LRT, WCON and HOPE. In fact, this correspondence can be made somewhat precise. Stalnaker (1965) demonstrated that his semantics is sound and complete with respect to the conditional logic $\mathrm{C} 2$ whose axioms are ID, CC, CA, CSO, CMon, MP, CS, $\mathrm{CN}_{1}$ and $\mathrm{CN}_{2}$. Theorem 3 tells us that as long as $t>\frac{2}{3}$, all of these axioms are guaranteed to be $t$-valid. And by Proposition 2, we already have good reason to require $t>\frac{2}{3}$, even in the setting in which we consider only ordinary truth functional propositions and ignore indicative conditionals. So we can accept every valid conditional inference of the Stalanker semantics without running into conflict with the conjunction of LT, RT, LRT, WCON and HOPE. ${ }^{23}$ This is in stark contrast with the material conditional and strict conditional semantics, both of which set us straight on a collision course with those principles. ${ }^{24}$

Clearly then, epistemologists with a stake in the epistemic norms LT, RT and WCON have a very good reason to accept the Stalnaker semantics as giving the correct picture of the logic of the indicative conditional. Since RT is the only one of these norms that specifically concerns the epistemology of indicative conditionals, this suggests that there is a clear and important sense in which the Stalnaker semantics describes the logic of the indicative conditional whose epistemology accords with RT. And in some sense, this is not surprising. For, in his initial presentation of the semantics, Stalnaker described himself as trying to make a 'transition' from the belief conditions for conditionals given by the Ramsey test to a corresponding set of truth conditions:

$[\mathrm{T}]$ he problem is to make the transition from belief conditions to truth conditions; that is, to find a set of truth conditions for statements having conditional form which explains

\footnotetext{
${ }^{22}$ Interestingly, CC is the only principle which is valid for all $t>\frac{2}{3}$, but not for all $t>r^{-1}$.

${ }^{23}$ Note also that the other rules which are $t$-valid for some admissible values of $t$ (RT and MT) are logically entailed by the conjunction of the axioms of $\mathrm{C} 2$.

${ }^{24}$ At this point, one might wonder what Theorem 3 means for existing probabilistic approaches to conditional logic. For example, Adams (1975) develops his own theory of conditional logic based on his famous probabilistic interpretation of the Ramsey test. Given that this logic is also based on the Ramsey test, one might expect it to fare well in the present context. However Adam's conditional logic, like the logic of the strict conditional, ends up both validating inferences (such as OIF) which are not $t$-valid for any admissible $t$, and also invalidating inferences (such as MT) which are $t$-valid for some admissible $t$. So the present analysis provides sufficient grounds to prefer Stalnaker's logic to Adams', despite the well noted similarity between the two systems.
} 
why we use the method we do use to evaluate them. The concept of a possible world is just what we need to make this transition, since a possible world is the ontological analogue of a stock of hypothetical beliefs. (Stalnaker 1968: 102)

Stalnaker explicitly conceived of his semantics as the natural translation of the Ramsey test into truth conditions for conditional statements (both subjunctive and indicative). In subsequent work, he also endorsed a probabilistic formalisation of the Ramsey test which came to be known as 'Stalnaker's thesis' (ST). According to ST, an agent's credence in the indicative conditional $\phi \rightarrow \psi$ should be identified with their conditional credence in $\psi$ given $\phi, c(\psi \mid \phi)$. So on Stalnaker's conception, the logic of the indicative conditional is given by the Stalnaker semantics, the epistemology of indicative conditionals is given by the probabilistic interpretation of the Ramsey test encoded in ST, and the logical and epistemological aspects cohere nicely.

Significant doubt was cast on this conception when Lewis (1976) proved his famed triviality results, which many authors accepted as a straightforward demonstration that ST could not possibly provide a viable account of the epistemology of indicative conditionals. And since Stalnaker himself formally interpreted the Ramsey test as ST, this appeared to demonstrate that the Ramsey test did not really cohere with the Stalnaker semantics in the way that Stalnaker hoped it would. What I've shown here is that there is a meaningful sense in which Stalnaker really was right about the close correspondence and mutual coherence between the Ramsey test and his semantics for the indicative. In particular, if one adopts the epistemic norms WCON, LT and the Lockean formalisation of RT given by LRT (as opposed to ST), then HOPE entails that the conditional logic corresponding to the Stalnaker semantics $(\mathrm{C} 2)$ is the strongest logic that one is licensed to assume for the indicative. 


\section{§5: Appendix}

\section{Proof of Theorem 3:}

I address each rule individually.

ID: Immediate from $c(\phi \mid \phi)=1>t$.

CS: Let $c(\phi \wedge \psi)=t$. Then $c(\psi \mid \phi)=\frac{c(\phi \wedge \psi)}{c(\phi)} \geq \frac{t}{c(\phi)} \geq t$.

$\mathbf{C N}_{1} / \mathbf{C N}_{2}$ : Choose arbitrary $t$ and suppose that $\phi \rightarrow \psi$ is disbelieved, i.e. $c(\psi \mid \phi) \leq 1-t$. Then $c(\neg \psi \mid \phi) \geq t$, meaning that $\phi \rightarrow \neg \psi$ is believed. Conversely, if $\phi \rightarrow \neg \psi$ is believed then $c(\neg \psi \mid \phi) \geq t$ and hence $c(\psi \mid \phi) \leq 1-t$, which implies that $\phi \rightarrow \psi$ is disbelieved, as desired.

MP: Let $t>r^{-1}, c(\psi \mid \phi), c(\phi) \geq t$. Then

$$
c(\psi) \geq c(\phi \wedge \psi)=c(\psi \mid \phi) b(\phi)>r^{-1^{2}}=1-r^{-1}>1-t
$$

Which implies $c(\neg \psi)<t$, as desired. Let $t<r^{-1}, c(\psi \mid \phi)=t=c(\phi)$ and $c(\neg \phi \wedge \psi)=0$. Then

$$
c(\psi)=c(\phi \wedge \psi)=c(\psi \mid \phi) b(\phi)<r^{-1^{2}}=1-r^{-1}<1-t
$$

Which implies $c(\neg \psi)>t$, as desired.

MT: Let $t>r^{-1}, c(\psi \mid \phi), c(\neg \psi) \geq t$. Then

$$
\begin{array}{r}
c(\phi)=c(\phi \mid \psi) c(\psi)+c(\phi \mid \neg \psi) c(\neg \psi) \\
\leq(1-t)+c(\phi \mid \neg \psi) c(\neg \psi) \\
=(1-t)+(1-c(\psi \mid \phi)) c(\phi) \\
\leq(1-t)+(1-t) c(\phi)
\end{array}
$$

Rearranging gives $c(\phi) \leq \frac{1-t}{t}$. And since $t>r^{-1}, \frac{1-t}{t}<t$, which implies $c(\phi)<t$, as desired. Let $t \leq r^{-1}$, and let $c(\phi \wedge \psi)=t^{2}, c(\phi \wedge \neg \psi)=t-t^{2}, c(\neg \phi \wedge \psi)=1-t^{2}-t, c(\neg \phi \wedge \neg \psi)=t^{2}$. It's 
easy to check that $c$ is probabilistic and $t=c(\psi \mid \phi)=c(\phi)=c(\neg \psi)$, i.e. $c(\phi) \geq t$, as desired.

RT: Let $c(\psi \mid \phi) \geq t, c(\xi \mid \phi \wedge \psi) \geq t$. Then for $t>r^{-1}$, we have

$$
\begin{array}{r}
c(\xi \mid \phi)=c(\xi \mid \psi \wedge \phi) c(\psi \mid \phi)+c(\xi \mid \phi \wedge \neg \psi) c(\neg \psi \mid \phi) \\
\geq c(\xi \mid \psi \wedge \phi) c(\psi \mid \phi) \\
\geq t^{2} \\
>1-t
\end{array}
$$

Conversely, let $t \leq r^{-1}$. Then the distribution given by $c(\phi \wedge \psi \wedge \xi)=t-t^{2}, c(\phi \wedge \psi \wedge \neg \xi)=t^{2}$, $c(\phi \wedge \neg \psi \wedge \neg \xi)=1-t$ satisfies the inequalities $c(\xi \mid \phi \wedge \psi)=\frac{t-t^{2}}{t^{2}} \geq t, c(\psi \mid \phi)=t$ and $c(\xi \mid \phi)=t-t^{2} \leq 1-t$.

CSO: Let $c(\psi \mid \phi), c(\phi \mid \psi), c(\xi \mid \phi) \geq t$. Then we have

$$
\begin{array}{r}
(i) c(\xi \mid \psi)=c_{\psi}(\xi \wedge \phi)+c_{\psi}(\xi \wedge \neg \phi) \\
=c_{\psi}(\xi \mid \phi) c_{\psi}(\phi)+c_{\psi}(\xi \mid \neg \phi) c_{\psi}(\neg \phi) \\
=c(\xi \mid \phi \wedge \psi) c(\phi \mid \psi)+c(\xi \mid \neg \phi \wedge \psi) c(\neg \phi \mid \psi)
\end{array}
$$

$(i i) t \leq c(\xi \mid \phi)=c(\xi \mid \phi \wedge \psi) c(\psi \mid \phi)+c(\xi \mid \phi \wedge \neg \psi) c(\neg \psi \mid \phi)$

From (ii), we get

$$
\begin{aligned}
& (i i i) t \leq c(\xi \mid \phi \wedge \psi) c(\psi \mid \phi) \\
& =c(\xi \mid \phi \wedge \psi) \frac{c(\phi \mid \psi) c(\psi)}{c(\phi)}
\end{aligned}
$$


And hence

$$
\begin{array}{r}
(i v) c(\xi \mid \phi \wedge \psi) c(\phi \mid \psi) \geq t \frac{c(\phi)}{c(\psi)} \\
\geq t \frac{c(\phi \wedge \psi)}{c(\psi)} \\
=t c(\phi \mid \psi) \\
\geq t^{2}
\end{array}
$$

Together with (i), this entails $c(\xi \mid \psi) \geq t^{2}$, and hence that $c(\xi \mid \psi)>1-t$ for $t>r^{-1}$. Conversely, let $t<r^{-1}$. Then the distribution given by $c(\phi \wedge \psi \wedge \xi)=\frac{t}{2}=c(\phi \wedge \psi \wedge \neg \xi), c(\phi \wedge \neg \psi \wedge \xi)=t^{2}$, $c(\neg \phi \wedge \psi \wedge \neg \xi)=1-t-t^{2}$ is probabilistic and it follows that $c(\psi \mid \phi)=\frac{t}{t+t^{2}}>t, c(\phi \mid \psi)=\frac{t}{1-t^{2}}>t$, $c(\xi \mid \phi)=\frac{\frac{t}{2}+t^{2}}{t+t^{2}}>t, c(\xi \mid \psi)=\frac{\frac{t}{2}}{1-\frac{t}{2}}<t$.

CC: Let $c(\psi \mid \phi), c(\xi \mid \phi) \geq t$, i.e. $\quad c_{\phi}(\psi), c_{\phi}(\xi) \geq t$. This implies that $c_{\phi}(\psi \vee \xi)=$ $c_{\phi}(\psi)+c_{\phi}(\xi)-c_{\phi}(\psi \wedge \xi) \geq 2 t-c_{\phi}(\psi \wedge \xi)$. For $t>\frac{2}{3}$, this entails $c_{\phi}(\psi \wedge \xi)>1-t$. Conversely, let $t \leq \frac{2}{3}$. Then the conditional distribution defined by $\frac{t}{2}=c_{\phi}(\psi \wedge \xi)=c_{\phi}(\psi \wedge \neg \xi)=c_{\phi}(\neg \psi \wedge \xi)$, $c_{\phi}(\neg \psi \wedge \neg \xi)=1-\frac{3 t}{2}$ is probabilistic and satisfies $c(\psi \mid \phi), c(\xi \mid \phi) \geq t, c_{\phi}(\psi \wedge \phi) \leq 1-t$, as desired.

CA: Let $c(\xi \mid \phi), c(\xi \mid \psi) \geq t, c(\xi \mid \phi \vee \psi) \leq 1-t$. To simplify the calculations, let

$$
\begin{gathered}
c(\phi \wedge \psi \wedge \xi)=u, c(\phi \wedge \psi \wedge \neg \xi)=v \\
c(\phi \wedge \neg \psi \wedge \xi)=w, c(\phi \wedge \neg \psi \wedge \neg \xi)=x \\
c(\neg \phi \wedge \psi \wedge \xi)=y, c(\neg \phi \wedge \psi \wedge \neg \xi)=z
\end{gathered}
$$

Note first that the assumptions imply the following inequalities,

$$
\text { (i) } u+w+y \leq 1-t \text {, (ii) } \frac{u+v}{u+v+w+x} \geq t \text {, (iii) } \frac{u+y}{u+v+y+z} \geq t
$$

From (i), we get

$$
\text { (iv) } u+y \leq(1-t)-w
$$

With (iii), this entails 


$$
\begin{gathered}
\frac{1-t-w}{u+v+y+z} \geq t \\
\frac{1-t}{t} \geq u+v+\frac{w}{t}+y+z . \\
\frac{1-t}{t} \geq 1-x-w+\frac{w}{t} . \\
x \geq 1-\left(\frac{1-t}{t}\right)-w+\frac{w}{t} . \\
\text { (v) } x \geq 2-\frac{1}{t}+\frac{w}{t}-w \geq 2-\frac{1}{t} .
\end{gathered}
$$

Combined with (i) and (ii), this yields

$$
\begin{aligned}
t \leq & \frac{u+w}{u+v+w+x} \\
\leq & \frac{u+w}{u+w+x} \\
& \leq \frac{1-t}{1-t+x} \\
& \leq \frac{1-t}{1-t+x} \\
\leq & \frac{1-t}{1-t+2-\frac{1}{t}}
\end{aligned}
$$

And $t>r^{-1}$ implies $\frac{1-t}{1-t+2-\frac{1}{t}} \leq \frac{1}{2}$, implying that $t<\frac{1}{2}$. So $t \leq r^{-1}$, as desired. Conversely, let $t \leq r^{-1}$ and consider the distribution given by $u=\frac{1-t}{t}, \frac{2 t-1-t^{2}}{2 t}=w=y, \frac{t}{2}=x=z$. By definition, $u+w+y \leq 1-t$ and hence $c(\xi \mid \phi \vee \psi) \leq 1-t$. Furthermore, $\frac{u+w}{u+v+w+x}=\frac{u+w}{u+w+x}=\frac{\frac{1-t}{t}+\frac{2 t-1-t^{2}}{2 t}}{\frac{1-t}{t}+\frac{2 t-1-t^{2}}{2 t}+\frac{t}{2}}$, and $\frac{\frac{1-t}{t}+\frac{2 t-1-t^{2}}{2 t}}{\frac{1-t}{t}+\frac{2 t-1-t^{2}}{2 t}+\frac{t}{2}} \geq t$ for all $t \leq r^{-1}$. So for $t \leq r^{-1}, c(\xi \mid \phi) \geq t$, and similarly for $c(\xi \mid \psi)$, as desired.

CMon: Let $c(\xi \mid \phi), c(\psi \mid \phi) \geq t$ and suppose that $c(\xi \mid \phi \wedge \psi) \leq 1-t$. To simplify the calculations, let $x=c(\xi \wedge \phi \wedge \psi), y=c(\phi \wedge \psi \wedge \neg \xi), z=c(\phi \wedge \neg \psi \wedge \xi)$. The assumptions ensure that $x+y, x+z \geq t$ and $\frac{x}{x+y} \leq 1-t$. Some simple algebra then gives us the following inequalities

$$
\text { (i) } z \leq 1-x-y \text {, (ii) } x \geq t-z \text {, (iii) } x \leq \frac{(1-t) y}{t}
$$

Since $x+y, x+z \geq t$, we also get $1 \geq x+y+z \geq 2 t-x$, and hence

$$
\text { (iv) } x \geq 2 t-1
$$

Together with (iii), (iv) implies 


$$
\text { (v) } y \geq \frac{t(2 t-1)}{1-t}
$$

Combined with $(i)$, this gives us

$$
\text { (vi) } z \leq 1-\left(2 t-1+\frac{t(2 t-1)}{1-t}\right)=2-2 t-\frac{t(2 t-1)}{1-t}
$$

With (ii), this implies

$$
\text { (vii) } x \geq 3 t-2+\frac{t(2 t-1)}{1-t}
$$

Combined with (v) and (i), this then yields

$$
\text { (viii) } z \leq 3-3 t-\frac{2 t(2 t-1)}{1-t}
$$

Now, the steps from (vi) to (viii) can be iterated to derive the following inequality for any $n \geq 2$

$$
\text { (ix) } z \leq n-n t-\frac{(n-1) t(2 t-1)}{1-t}
$$

And it turns out that for any $t>r^{-1}$, there exists some $n$ such that the term on the right hand side of (ix) is negative. Since $c$ is probabilistic, this entails that $t \leq r^{-1}$, as desired. Conversely, let $t \leq r^{-1}$. Then the distribution given by $c(\phi \wedge \psi \wedge \xi)=t-t^{2}, c(\phi \wedge \psi \wedge \neg \xi)=t_{2}=c(\phi \wedge \neg \psi \wedge \xi)$, $c(\neg \phi \wedge \neg \psi \wedge \neg \xi)=1-t-t^{2}$ is probabilistic, and it satisfies $c(\xi \mid \phi \wedge \psi)=1-t, c(\psi \mid \phi)=t=c(\xi \mid \phi)$, as desired.

Cont: Let $c(\phi \wedge \psi)=t, c(\phi \wedge \neg \psi)=1-t$. Then $c(\psi \mid \phi)=t, c(\neg \phi \mid \neg \psi)=0$.

NA: Let $c(\phi \wedge \neg \psi)=1-t, c(\neg \phi \wedge \psi)=t$. It's easy to see that $c$ satisfies $c(\neg \phi)=t$, $c(\psi \mid \phi)=0<t$ as desired.

HS: That HS is not $t$-valid for any $t$ follows from the observation that $c(\xi \mid \psi)$ and $c(\psi \mid \phi)$ don't constrain $c(\xi \mid \phi)$ at all, as long as $c(\xi \mid \psi)$ and $c(\psi \mid \phi)$ aren't both 1. For example, consider an $n$-sided die for arbitrarily large $n$. Let $\phi$ be 'I roll a 1', let $\psi$ be 'I roll any number' and let $\xi$ be 'I roll a number greater than 1'. Then $c(\psi \mid \phi)=1, c(\xi \mid \psi)=\frac{n-1}{n}$ and $c(\xi \mid \phi)=0$.

SA: Let $c(\phi \wedge \neg \psi \wedge \xi)=1-t, c(\phi \wedge \psi \wedge \neg \xi)=t$. It's easy to see that $c$ satisfies $c(\psi \mid \phi)=t$, $c(\psi \mid \phi \wedge \xi)=0<t$ as desired. 
TC: Let $c(\neg \phi \wedge \psi)=t, c(\phi \wedge \neg \psi)=1-t$. Then $c(\psi) \geq t$ and $c(\psi \mid \phi)=0$.

OIF: Let $c(\phi \wedge \psi)=t, c(\neg \phi \wedge \neg \psi)=1-t$. Then $c(\phi \vee \psi) \geq t$ and $c(\psi \mid \neg \phi)=0$.

RCM: Let $c(\phi \wedge \psi \wedge \neg \xi)=1-t, c(\neg \phi \wedge \neg \psi \wedge \neg \xi)=t$, which satisfies $c(\psi \supset \xi) \geq t$. Then $c(\psi \mid \phi)=1$ and $c(\xi \mid \phi)=0<t$.

RCR: Let $c(\phi \wedge \psi \wedge \xi \wedge \neg \gamma)=1-t, c(\neg \phi \wedge \neg \psi \wedge \neg \xi \wedge \neg \gamma)=t$. Then $c((\psi \wedge \xi) \supset \gamma) \geq t$, $c(\psi \mid \phi)=c(\xi \mid \phi)=1$ and $c(\gamma \mid \phi)=0<t$.

RCEA Let $c(\neg \phi \wedge \neg \psi \wedge \neg \xi)=t, c(\phi \wedge \neg \psi \wedge \xi)=\frac{1-t}{2}=c(\neg \phi \wedge \psi \wedge \neg \xi)$. Then $c(\phi \equiv \psi)=t$, $c(\xi \mid \phi)=1$ and $c(\xi \mid \psi)=0$.

RCEC: Let $c(\phi \wedge \psi \wedge \neg \xi)=1-t, c(\neg \phi \wedge \psi \wedge \xi)=\frac{t}{2}=c(\neg \phi \wedge \neg \psi \wedge \neg \xi)$. Then $c(\psi \equiv \xi)=t$, $c(\psi \mid \phi)=1, c(\xi \mid \phi)=0$.

CCO: Let $\psi=\neg \phi, \xi=\top$ and suppose $c(\phi)=1-t$. It trivially follows that $c(\psi \mid \xi)=t$, $c(\xi \mid \psi)=1, c(\xi \mid \phi)=1$ and $c(\psi \mid \phi)=0$.

\section{References}

Adams, Ernest. (1975). The logic of conditionals: An application of probability to deductive logic, volume 86. Springer.

Bradley, Richard. (2007). A Defence of the Ramsey Test. Mind, 116(461): 1-21.

Clarke, Roger. (2013). Belief is Credence One. Philosopher's Imprint, 13(11): 1-18.

Douven, Igor. (2015). The Epistemology of Indicative Conditionals. Cambridge: Cambridge University Press. 
Eva, Benjamin., Shear, Ted. and Fitelson, Branden. (Unpublished). Four Approaches to Supposition.

Foley, Richard. (1992). The Epistemology of Belief and the Epistemology of Degrees of Belief. American Philosophical Quarterly, 29(2): 111-124.

Foley, Richard. (2000). Working Without a Net. Oxford: Oxford University Press.

Foley, Richard. (2009). Beliefs, degrees of belief, and the Lockean thesis. In: F. Huber, and C. Schmidt-Petri (Eds.), Degrees of belief, Synthese Library 342. Springer.

Hanson, Sven Ove. (1992). In Defence of the Ramsey Test. Journal of Philosophy, 89(10): $522-540$.

Hartmann, Stephan. and Stern, Reuben. (Forthcoming). Two Sides of Modus Ponens. Journal of Philosophy.

Hawthorne, James. and Bovens, Luc. (1999). The preface, the lottery, and the logic of belief. Mind, 108(430): 241-264.

Jackson, Frank. (1979). On Assertion and Indicative Conditionals. Philosophical Review, 88: $565-589$.

Knight, Kevin. (2002). Measuring Inconsistency. Journal of Philosophical Logic, 31(1): 77-98.

Kyburg, Henry. (1961). Probability and the Logic of Rational Belief. Middletown, CT: Wesleyan University Press.

Leitgetb, Hannes. (2017). The Stability of Belief. Oxford: Oxford University Press.

Lewis, Clarence Irvin. (1918). A Survey of Symbolic Logic. University of California Press. 
Lewis, David. (1973) Counterfactuals. Cambridge, MA: Harvard University Press.

Lewis, David. (1976). Probabilities of Conditionals and Conditional Probabilities. Philosophical Review, 85(3): 297-315.

McGee, Vann. counterexample to modus ponens. Journal of Philosophy, 82 (9): 462-471.

Shear, Ted. and Fitelson, Branden. (2018). Two Approaches to Belief Revision. Erkenntnis, https://doi.org/10.1007/s10670-017-9968-1

Sorensen, Roy. (2006). Epistemic Paradoxes. Stanford Encyclopedia of Philosophy.

Stalnaker, Robert. (1968). A Theory of Conditionals. In Nicholas Rescher (ed.), Studies in Logical Theory (American Philosophical Quarterly Monographs 2). Oxford: Blackwell. 98-112.

Stalnaker, Robert. and Thomas, Richmond. (1970). A Semantic Analysis of Conditional Logic. Theoria, 36(1): 23-42.

Starr, Will. (unpublished). Indicative Conditionals, Strictly.

Yalcin, Seth. (2012). A Counterexample to Modus Tollens. Journal of Philosophical Logic, 41(6): 1001-1024. 